\title{
Consultation conjointe médico-psychologique (CCMP) : étude préliminaire de follow-up auprès de 13 patientes souffrant de paresthésies buccales médicalement inexpliquées
}

\author{
Joint medical and psychological consultation (JMPC): preliminary follow-up \\ study with 13 patients with burning mouth syndrome
}

MARLÈNE SARTORI*, CORINNE ROSSET ${ }^{* *}$, COLETTE DEGIVE* ${ }^{*}$ VÉRONIQUE HAYNAL*, CHRISTINE CUNIER-DROCCO**

\section{RÉSUMÉ}

La consultation conjointe médico-psychologique (CCMP) constitue un mode de prise en charge des patientes souffrant de paresthésies buccales médicalement inexpliquées. Une étude préliminaire de follow-up a été réalisée dans le but de déterminer les variables à mesurer pour évaluer l'efficacité de cette technique de prise en charge.

L'analyse des résultats suggère de poursuivre le recueil des auto-évaluations et des hétéro-évaluations après les CCMP, et d'investiguer l'état somatique, l'état psychique, les acquisitions faites pendant les CCMP, l'utilité des CCMP et l'évolution de la consommation médicale des patientes. Mais aussi de donner plus de poids à la fonction de rassurer et d'expliquer. (Med Buccale Chir Buccale 2006; 12: 31-38).

mots clés : consultation conjointe médico-psychologique, paresthésies buccales médicalement inexpliquées, étude préliminaire de follow-up, efficacité.

\section{SUMMARY}

Patients with burning mouth syndrome visit a joint medical and psychological consultation (JMPC). The JMPC constitutes a manner of handling patients suffering from burning mouth syndrome.

A preliminary follow-up study was done to determine the variables that have to be measured in order to evaluate the efficacity of this patient handing technique. The analysis of the results suggests that we must continue collecting the auto-evaluations and hetero-evaluations after the JMPC and to investigate into the somatic state, the psychic state, the data acquired during the JMPC, the usefulness of the JMPC and the evolution of the medical consumption of the patients, but also in giving more weight to the function of reassuring and explaining. (Med Buccale Chir Buccale 2006; 12: 31-38).

key words: joint medical and psychological consultation, burning mouth, syndrome, preliminary follow-up study, efficacity.

\footnotetext{
* Unité de psychiatrie de liaison, SAULP, Hôpitaux Universitaires de Genève, Suisse

** Division de stomatologie et chirurgie orale, Faculté de Médecine de Genève, Suisse
}

Demande de tirés à part :

Christine Cunier-Drocco Division de Stomatologie Chirurgie Orale et Radiologie Dento-Maxillo-Faciale rue Barthélémy-Menn 191211 Genève 4 Suisse christine.cunier@medecine.unige.ch 
médecine

buccale

chirurgie

buccale

VOL. $12, \mathrm{~N}^{\circ} 1$ 2006

page 32
La consultation conjointe médico-psychologique (CCMP) est une technique de prise en charge qui diffère de la prise en charge habituelle [4]. Dans le référé classique, lorsqu'un médecin connaît des difficultés de relation avec un patient, ou ne comprend pas certains aspects de sa plainte, il recourt à un collègue psychologue ou psychiatre en lui disant: " Je voudrais vous adresser tel patient... » ou «Donner moi votre avis sur ce patient... ». Le point de départ de la technique de la CCMP se trouve dans la réponse donnée par le psychologue ou le psychiatre au médecin: "Je préfère exercer devant vous et me rendre compte sur place de ce qui est en jeu pour vous et pour le patient... ». La CCMP est donc menée en binôme et met à jour, au fil des consultations, les aspects psychosociaux de l'affection, en même temps que les aspects médico-chirurgicaux, dans le but d'apporter au malade la meilleure aide possible. Depuis 1997, une collaboration de ce type existe entre le service de stomatologie et chirurgie orale de la Faculté de Médecine de Genève et l'unité de psychiatrie de liaison, unité à l'origine de ce type de consultations dans d'autres services des Hôpitaux Universitaires de Genève [5,9]. Cette prise en charge conjointe en stomatologie s'adresse aux patients souffrant de paresthésies buccales médicalement inexpliquées (PBMI) [11]: sensation de brûlures, picotements, etc., présents dans la sphère buccale sans lésions organiques objectivables [8]. La CCMP permet d'apporter à ces patients un soulagement, voire la disparition des symptômes, ainsi qu'un début de prise de conscience du sens de leur plainte. En proposant aux patients de s'exprimer spontanément lorsqu'ils décrivent leur plainte ou ce qu'ils ressentent, on observe un investissement de leur part vers les thérapeutes. Un dialogue à trois s'instaure alors de manière plus souple et régulière. Cette CCMP est tenue par une odontostomatologiste et une psychologue et se déroule dans le service de stomatologie et chirurgie orale. Elle répond aux 6 principes de base des consultations conjointes [6]:

1. La CCMP constitue une prise en charge globale du patient.

2. La CCMP implique, par définition, la présence simultanée des deux thérapeutes.
3. Une complicité doit exister entre les thérapeutes dès la constitution du duo.

4. La CCMP requiert des connaissances théoriques des domaines réciproques des deux thérapeutes.

5. Les rôles et les responsabilités des deux thérapeutes ne sont pas interchangeables.

6. Le praticien dont le champ de connaissances répond le mieux à ce dont le patient a besoin en priorité clôture la consultation.

Depuis 1997, 260 patients ont été suivis à cette consultation. Depuis 2003, un second tandem odontostomatologiste-psychologue effectue également une CCMP. Une étude pilote de follow-up a été mise en route pour vérifier que la prise en charge globale de type bi-focal est une technique abordable pour cette population anxieuse et découragée par son parcours médical antérieur et complexe. Deux cas sont présentés brièvement ci-dessous pour illustrer le fonctionnement de la CCMP et les principales caractéristiques des patients pris en charge.

Cas 1 : Il s'agit d'une patiente, proche de la soixantaine, qui vient en CCMP avec une plainte à type de brûlures sur la pointe de la langue présente depuis 3 mois. Elle fait part d'une situation familiale assez complexe qui est la source de beaucoup de fatigue (maladie d'Alzheimer chez les beaux-parents, fille ex-toxicomane, parents âgés, etc.). Au cours de la seconde consultation, la patiente montre beaucoup de collaboration et de désir pour être très présente dans cette relation à trois. Des moments privilégiés peuvent être mentionnés: diminution des symptômes de brûlures et prise de conscience à un moment bref et précis de ce que peut être une difficulté psychologique... II n'y a donc pas eu, bien au contraire, de réorganisation défensive, et la patiente a pu réaliser une levée presque totale des symptômes, accompagnée d'idées conscientes sur tout ce qui la gênait ou la préoccupait de façon sous-jacente. Ce cas clinique illustre un aspect des CCMP: plus les patients consultent tôt après l'apparition des symptômes, plus ils bénéficieront de l'aide apportée. Quatre séances ont été nécessaires et suffisantes pour lui faire vivre une autre perspective d'elle-même et de ses problèmes. 
Le pronostic en consultation est bon, tout comme le pronostic dans le follow-up. Réalisé 5 ans après les CCMP, le follow-up confirme d'une part la diminution, voire la disparition, de la symptomatologie et d'autre part, la capacité nouvelle de la patiente à appréhender sa vie psychique, par exemple réaliser une auto-analyse lorsque des picotements surgissent. II renseigne également sur la richesse du lien établi avec elle lors des séances.

Cas 2: Cette patiente de 64 ans est adressée par une odontostomatologiste. Le diagnostic de PBMI est établi mais ce n'est deux ans plus tard que la patiente se présente à la CCMP. Le setting proposé est accepté d'emblée et, lors de la première consultation, deux éléments retiennent l'attention: d'une part, la description de sensation de brûlures sur la langue et, d'autre part, le récit d'un événement vécu quelques années auparavant (séjour en Allemagne pour l'inhumation de sa belle-mère et participation lors de ce séjour au carnaval local avec son mari). La patiente fait ce récit avec émotion en mettant en évidence le côté paradoxal de la juxtaposition de ces deux événements. La patiente associe à cet épisode l'origine de ses manifestations buccales. II faut relever aussi que la patiente confie une déception conjugale profonde remontant au début du mariage. Lors de la $2^{\mathrm{e}}$ consultation, les deux éléments suivants sont retenus:

- tentative de modification de sa vie quotidienne; - sentiment de culpabilité et affect dévalorisant induits par le mari et, très probablement, par la patiente également.

C'est avec une forme de plaisir que la patiente fait elle-même la prise de conscience que la situation buccale et la situation affective sont liées. Le mieux-être buccal est réel, le soulagement est tel que la patiente décide de mettre fin aux CCMP. Six mois plus tard, la patiente revient à la CCMP et parle de son émancipation, du lien entre anxiété et brûlures, du sentiment de tristesse qui a disparu et de son réel mieux-être.

Lors du follow-up réalisé six ans après les CCMP, on considère que le souvenir de ces consultations est intact chez cette patiente. La prise de conscience reste le moteur de tout changement qu'elle souhaite apporter dans sa vie. La modifi- cation du sentiment de culpabilité dans le sens d'un allègement est presque total. Son estimation de l'utilité des CCMP est exprimée ainsi : «C'est bien si les gens ont cette exigence de se confier».

Le bénéfice de la CCMP devrait résider en une modification intra-personnelle et en une modification en relation avec des facteurs externes [7]. Le but de l'étude préliminaire de follow-up consiste à chercher des variables opérationnelles pour vérifier que cette technique est abordable en comparant notre hétéro-évaluation avec l'autoévaluation des patientes. Les résultats de cette étude de follow-up sont présentés et commentées ci-dessous.

\section{MATERIEL ET MÉTHODE}

Les paramètres pris en compte pour évaluer les modifications au niveau intra-personnel sont l'état somatique et l'état psychique des patientes, ainsi que leurs acquisitions pendant les CCMP [14]. Les paramètres permettant d'explorer les facteurs externes sont l'utilité perçue des CCMP et l'évolution de la consommation médicale.

La mise à l'épreuve de l'hypothèse initiale est faite sur la base des variables suivantes:

- L'état somatique est jugé à partir de la fréquence et/ou de l'intensité des symptômes. Une diminution de leur fréquence et/ou de leur intensité est assimilée à une amélioration.

- L'état psychique est évalué par le degré d'inquiétude face aux symptômes (une amélioration se traduisant par une diminution de l'inquiétude) et/ou le mieux-être en général [13].

- Les acquisitions faites pendant les CCMP sont investiguées. Une vérification est faite en demandant aux patientes si elles savent qu'elles ne sont pas les seules à avoir ces symptômes.

- L'évaluation de l'utilité des CCMP est réalisée à partir des appréciations données par les patientes: utilité pour elle-même, pour d'autres patients et pour d'autres symptômes. Chaque élément est codé (0 à 3 ), la somme doit être égale ou supérieure à 5 pour que l'utilité soit jugée positive.

- La consommation médicale est évaluée à partir de la fréquence des consultations médicales médecine

buccale

chirurgie

buccale

VOL. $12, \mathrm{~N}^{\circ} 1$ 2006

page 33 
médecine

buccale

chirurgie

buccale

VOL. $12, \mathrm{~N}^{\circ} 1$ 2006

page 34 où la plainte de PBMI a été évoquée (1 à 2 fois/an étant estimée comme normal).

En conclusion, on considère que les CCMP ont profité aux patientes si :

- une amélioration de l'état somatique et/ou de

l'état psychique est constatée,

-et si l'évaluation des CCMP par les patientes ou de leur consommation médicale est positive.

L'hétéro-évaluation et l'auto-évaluation sont réalisées en analysant respectivement l'attente des praticiens (selon les critères déterminés dans l'hypothèse de départ) par rapport à l'attente des patientes (question posée à la fin de l'entretien de follow-up). L'évaluation se termine par le passage de tests (QDSA, HAD, changements psychosociaux...) afin d'apprécier leur pertinence comme outils d'évaluation avant et après traitement.

\section{Population, procédure et matériel}

26 sujets ont été sélectionnés à partir des 148 dossiers de patients suivis en CCMP entre 1997 et 2001. Les critères de choix furent:

- diagnostic de PBMI,

- sexe féminin,

- dernier rendez-vous remontant à plus d'un an,

- avec un nombre minimum de deux consultations,

- sélection au hasard de 8 dossiers par année entre 1999 à 2001, plus 1 cas dans les deux premières années (1997-1998).

Sur les 26 lettres envoyées, 7 sont revenues en raison d'un changement d'adresse et il y a eu 4 refus exprimés par téléphone. Au total, 15 patientes ont accepté de revenir pour une évaluation des CCMP. Parmi ces 15 patientes, 2 ont été exclues à posteriori, l'une en raison de troubles de la mémoire et l'autre pour des comorbidités somatiques.

Ces 13 patientes sont domiciliées dans le Canton de Genève (11) ou en France voisine (2). Huit d'entre elles sont mariées, 2 divorcées ou séparées, 2 veuves et 1 célibataire. La majorité (10/13) est de nationalité suisse mais d'origines variées: Espagne, Italie, Portugal, France, République Dominicaine...) et elles ont presque toutes (11/13) des enfants. Six patientes sont à l'âge de la retraite, 4 encore actives professionnellement et pour 3 cette donnée n'est pas précisée. L'âge moyen des patientes est de 58 ans (minimum
$=39$ ans, maximum = 81 ans). En moyenne, le nombre de consultations conjointes est de 4 pour chaque patiente.

L'entretien de follow-up est mené par le deuxième tandem de thérapeutes (odontostomatologistepsychologue) que les patientes ne connaissent pas. Le cadre de la CCMP du follow-up est le cadre habituel des CCMP, bien connu par les patientes (même salle d'attente, même cabinet dentaire, même disposition des 3 chaises...). Les thérapeutes participant à l'entretien n'auront jamais connaissance du dossier médical initial des patientes. L'entretien est structuré de façon strictement identique pour toutes les patientes: - Une première phase (phase libre) où la patiente est conviée à répondre comme elle l'entend à la question ouverte strictement identique pour toutes les patientes: "Comment allez-vous actuellement? ».

- Une deuxième phase (phase structurée) composée d'une suite de questions fermées concernant d'une part l'état somatique et psychique actuel de la patiente, et d'autre part son appréciation sur les acquis, le bénéfice et l'utilité des CCMP. - Entre les questions concernant l'état actuel de la patiente et son appréciation des CCMP, un examen dentaire de contrôle est effectué [1].

Après l'entretien, les patientes répondent à plusieurs questionnaires avec l'aide d'une autre psychologue. II s'agit de l'Echelle QDSA (Questionnaire de la Douleur de Saint Antoine; Boureau [1984]) ; Questionnaire HAD (Hospital Anxiety and Depression; Zigmond et Snaith [1983]). Elles répondent aussi à quelques questions de nature psychosociale (changements de travail, d'habitat, de structure familiale...) [2].

Le QDSA comprend 58 qualificatifs répartis en 16 classes:

- les 9 premières classes représentent l'aspect sensoriel de la sensation,

- les 7 dernières, l'aspect affectif,

- chaque classe comprend des adjectifs qui se traduisent par une note donnée par le patient (de 0 à 4).

La patiente choisit, parmi l'ensemble des 16 classes, l'adjectif qui correspond le mieux à sa gêne puis, dans un deuxième temps, elle lui attribue une note (de 0 à 4 ). 
Ce questionnaire d'auto-évaluation permet une quantification par un score total (QDSA-T) des aspects qualitatifs de la douleur (dans cette étude des PBMI) et permet de distinguer entre des qualifications sensorielles (sous-score QDSA-S) ou d'autres affectives (QDSA-A). Ce score semble ainsi traduire un vécu psychologique dépressif et/ou anxieux de la douleur. Le HAD est une échelle d'auto-évaluation qui permet de mesurer la symptomatologie actuelle de l'état affectif du patient. Elle est composée de 14 items dont la moitié évalue la dimension dépressive et l'autre moitié la dimension anxieuse. Un score supérieur à 10 pour une des 2 sous-échelles $D$ (dépression) ou $A$ (anxiété) indique la présence certaine d'une symptomatologie $\mathrm{D}$ et/ou A; entre 8 et 10, il s'agit d'un cas douteux.

Sur le plan psychosocial, on demande aux patients si, depuis la fin de la prise en charge, des événements importants ont eu lieu dans leur vie (mariages ou divorces dans la famille/départ ou arrivée de membres dans la famille/changement de lieu d'habitation ou changement professionnel, etc.). Après la récolte de quelques réponses spontanées, on se permet d'évoquer ou de vérifier certains autres changements éventuels. La patiente doit préciser comment elle juge chacun de ces évènements (positif, neutre ou négatif).

\section{Traitements des données}

L'entretien aboutit à une récolte de données de type qualitatif nominal ou ordinal (mieux/même chose/moins bien; ou encore positif/neutre/négatif...) qui seront réduites à des appréciations binaires (oui/non ou mieux/pas mieux correspondent respectivement à 1 ou 0), certaines pondérées par rapport aux années qui ont passé (consommation médicale et changements psychosociaux) selon la définition des hypothèses opérationnelles, pour aboutir à une évaluation finale du bénéfice (positif/négatif) apporté par la prise en charge effectuée dans les CCMP. Le bénéfice ainsi obtenu est comparé au jugement global des patientes pour obtenir une mesure des prétentions d'aboutissement thérapeutique. En effet, actuellement, on considère que le sentiment subjectif du patient, d'une manière générale, reprend de l'importance par rapport aux mesures dites objectives.
Le petit nombre de cas évalués dans cette étude ne permet pas de soumettre ces données à un contrôle statistique de plusieurs variables indépendantes, comme cela été prévu au départ. Ceci oblige à rechercher des concordances.

\section{PRÉSENTATION DES RÉSULTATS}

\section{Entretien de follow-up}

Pour plus des deux-tiers des patientes, le symptôme somatique est resté le même (9/13), mais il apparaît moins souvent et son intensité est moindre pour plus de la moitié de notre population (7-8/13) et, pour un cas, il a même complètement disparu. Pour plus de la moitié des patientes (9/13), la souffrance psychique a diminué et elle a disparu dans deux cas. L'inquiétude par rapport au symptôme a disparu pour presque la moitié des patientes $(6 / 13)$ et elle est moindre pour 4 autres patientes. Au total, 10 patientes sur 13 ont l'impression de se sentir mieux.

Pour environ deux tiers des patientes (9/13), la CCMP leur a appris (apporté) quelque chose (soutien, conseils, nouvelle vision de leur maladie, réflexion sur soi...). La moitié des patientes sont certaines de ne pas être seules à avoir souffert de cette pathologie (7/13) alors qu'un tiers ne s'est jamais posé la question (4/13). 8 patientes sur 13 ont jugé utiles les CCMP pour elles-mêmes, et 7 envisagent facilement l'hypothèse (7/13) que cela devrait être sûrement utile aussi pour d'autres personnes et pour d'autres affections similaires (6/13). 6 patientes $(6 / 13)$ n'ont pas eu besoin d'une prise en charge complémentaire pour leur PBMI ; après les CCMP, 5 autres patientes ont consulté 1 ou 2 fois pour leur PBMI (médecin généraliste, odontostomatologiste, psychiatre, ORL, neurologue, parodontologue). Pour 10 patientes sur 13, l'appréciation globale des CCMP est positive et pour 3 neutre. Aucune patiente n'a donné une évaluation négative des CCMP.

Les résultats obtenus pour chacune des variables sont convertis en variables nominales binaires où le 1 est favorable à l'hypothèse et le 0 défavorable à celle-ci.

Avec ces résultats (Tab. 1), on constate qu'il y a: - une amélioration de l'état somatique (Es) pour 9 patientes sur 13 , médecine

buccale

chirurgie

buccale

VOL. $12, \mathrm{~N}^{\circ} 1$ 2006

page 35 
- une amélioration de l'état psychique et des acquisitions à la CCMP (Epa) pour 8 patientes sur 13 ,

- une reconnaissance de l'utilité des CCMP (U) pour 7 patientes sur 13 ,

- une diminution de la consommation médicale (CM) pour 12 patientes sur 13.

Au niveau intra-personnel (Es/Epa) une amélioration est remarquée pour 9 des 13 patientes. Pour les facteurs externes (U/CM) une amélioration est constatée pour 12 patientes sur 13. Enfin, 9 patientes sur 13 ont obtenu une amélioration de leur état général (bio-psycho-social) selon les exigences fixées initialement (Fin). Les patientes considèrent dans 10 cas sur 13 avoir retiré un bénéfice des CCMP.

En ce qui concerne la comparaison de l'attente des patientes (PP) avec celles des thérapeutes (Fin), il existe une correspondance parfaite pour buccale

chirurgie

buccale

VOL. $12, \mathrm{~N}^{\circ} 1$ 2006

page 36
8 cas sur 13. Dans 2 cas, il semble que l'attente des praticiens est moins exigeante que celles des patientes ( $\mathrm{F} 1$ et $\mathrm{F} 7$ ); par contre, dans 3 autres cas, c'est l'inverse (F2, F4 et F10).

Lorsqu'on examine les concordances on relève qu'il y a une coïncidence:

- presque parfaite entre l'état somatique (ES) et l'état psychique et les acquisitions (EPA),

- parfaite entre l'état somatique (ES) et l'évaluation finale de l'amélioration bio-psycho-sociale (Fin égale 1 si Es/Epa et U/CM correspondent à 1),

- presque parfaite entre l'état psychique et les acquisitions (EPA) et l'évaluation finale (Fin égale 1 si Es/Epa et U/CM sont 1),

- parfaite entre l'état somato-psychique (intrapersonnel) du patient (ES/EPA) et l'évaluation finale (Fin égale 1 si Es/Epa et U/CM sont 1).

\section{Questionnaires}

Au QDSA, 5 patientes ont un sous-score affectif (A) comparable au sous-score sensoriel (S), 5 patientes ont un $A>S$ et 3 patientes ont un $\mathrm{S}>\mathrm{A}$. Par ailleurs, un lien significatif $(\mathrm{p}=.022)$ a été mis en évidence entre le sous-score QDSA$S$ et l'utilité des CCMP $(U)$ : les patientes qui considèrent les CCMP comme utiles (pour ellesmêmes et les autres ou pour d'autres symptômes) ont des scores plus élevés au QDSA-S [2]. Au
HAD, la majorité des patientes (10/13) ont un $A>D$, pour un quart des patientes le score d'anxiété (A) est plus ou moins égal au score de dépression (D). Ainsi, dans cette population, A est supérieur ou proche de D. Quatre patientes (F1, $F 4, F 11$ et F13) ont un score qui indique une symptomatologie anxieuse et 2 autres (F2 et F13) un score indiquant une symptomatologie dépressive. Et celles qui déclarent avoir eu des changements neutres au niveau psychosocial, ont un score HAD-D plus élevé ( $p=.011)[2,12,15]$.

Les patientes rapportent 31 changements psychosociaux survenus dans leur vie depuis la fin des CCMP, la moitié (17/31) de ces changements sont considérés comme positifs, 5 comme neutres et 9 comme négatifs. Six patientes sur 13 déclarent des événements négatifs et 1 seule (F10) une majorité d'événements négatifs (2/2). Comme cela a déjà été signalé ci-dessus, les patientes qui déclarent avoir des changements neutres, ont un score HAD-D plus élevé $(p=.001)$ [2].

\section{DISCUSSION}

On doit d'abord se demander si les variables investiguées répondent bien aux exigences. La comparaison hétéro-évaluation et auto-évaluation sur l'ensemble des patientes montre une correspondance assez moyenne ( $8 / 13$ soit $61 \%)$. II y a 5 patientes dont les résultats interpellent: 2 ont une auto-évaluation négative malgré un résultat presque totalement positif aux 4 variables, alors que les 3 autres ont des résultats presque totalement négatifs mais une auto-évaluation positive. Les 2 premières avaient probablement une attente plus élevée que ce que la CCMP pouvait leur offrir (du type "attente magique "); les résultats pour les 3 autres font envisagé les hypothèses suivantes: la cohérence et la fiabilité de leurs réponses, ainsi que la pertinence de nos questions. En reprenant en détail les réponses de ces 3 patientes, on constate que 2 n'ont pas eu d'amélioration de leurs facteurs intra-personnels, excepté le fait qu'elles aient été rassurées sur l'absence de gravité de leur affection. Elles ont donc compris que cette pathologie est connue, qu'elle 
Tableau 1 : Evaluations obtenues lors de l'entretien de follow-up (Es : état somatique ; Epa : état psychique et acquisition aux CCMP ; U : utilité des CCMP ; CM : consommation médicale ; Fin : évaluation finale ; BP : bénéfice selon le patient)

Follow-up assessment (Es : physical state ; Epa : psychological and knowledge ; $U$ : utility ; CM : consumption of medical advices ; BP : patient profit)

\begin{tabular}{|c|c|c|c|c|c|c|c|c|}
\hline \multirow[t]{2}{*}{$\mathrm{N}=13$} & \multicolumn{7}{|c|}{ Hétéro-évaluation } & \multirow{2}{*}{$\begin{array}{c}\text { Auto- } \\
\text { évaluation }\end{array}$} \\
\hline & mieux $=1$ & pas mieux $=0$ & $>5=1$ & $<2=1$ & $1 / 2=1$ & $1 / 2=1$ & $2 / 2=1$ & \\
\hline Code & Es & Epa & $U$ & CM & Es/Epa & $\mathrm{U} / \mathrm{CM}$ & Fin & BP \\
\hline F1 & 1 & 1 & 1 & 1 & 1 & 1 & 1 & 0 \\
\hline $\mathrm{F} 2$ & 0 & 0 & 0 & 0 & 0 & 0 & 0 & 1 \\
\hline F3 & 0 & 0 & 0 & 1 & 0 & 1 & 0 & 0 \\
\hline $\mathrm{F} 4$ & 0 & 0 & 1 & 1 & 0 & 1 & 0 & 1 \\
\hline F5 & 1 & 1 & 1 & 1 & 1 & 1 & 1 & 1 \\
\hline F6 & 1 & 1 & 1 & 1 & 1 & 1 & 1 & 1 \\
\hline $\mathrm{F} 7$ & 1 & 1 & 0 & 1 & 1 & 1 & 1 & 0 \\
\hline F9 & 1 & 1 & 0 & 1 & 1 & 1 & 1 & 1 \\
\hline F10 & 0 & 0 & 0 & 1 & 0 & 1 & 0 & 1 \\
\hline F11 & 1 & 1 & 1 & 1 & 1 & 1 & 1 & 1 \\
\hline F12 & 1 & 1 & 1 & 1 & 1 & 1 & 1 & 1 \\
\hline F13 & 1 & 1 & 1 & 1 & 1 & 1 & 1 & 1 \\
\hline F15 & 1 & 0 & 0 & 1 & 1 & 1 & 1 & 1 \\
\hline TOT & 9 & 8 & 7 & 12 & 9 & 12 & 9 & 10 \\
\hline$\%$ & 70 & 61 & 54 & 92 & 70 & 92 & 70 & 77 \\
\hline
\end{tabular}

médecine

buccale

chirurgie buccale

VOL. $12, \mathrm{~N}^{\circ} 1$ 2006

page 37 n'est pas grave, qu'elles ne sont pas les seules à en souffrir, et qu'il n'y a pas de prise en charge bien codifiée. Par contre, la troisième patiente n'est pas rassurée, elle croît être seule à souffrir de ses symptômes et continue à chercher une solution médicale qui fait défaut. Néanmoins, elle déclare que les CCMP ont été positives, mais qu'elle était et reste négative parce qu'il n'y a pas «de miracle». Ainsi, il semble que ni la cohérence, ni la fiabilité des réponses des patientes ne soient mises en cause. Par contre, il faut constater qu'il y a des patientes qui ont moins d'exigences thérapeutiques par rapport aux CCMP que celles des thérapeutes, et d'autres qui, malgré leur insatisfaction concernant l'évolution de leur symptôme, apprécient le cadre offert par la CCMP.

Par ailleurs, les résultats obtenus par l'hétéro-évaluation mettent en évidence un lien privilégié entre l'évaluation finale (Fin) et les facteurs intra-personnels (Es/Epa) par rapport aux facteurs externes (U/CM). En effet, la coïncidence est parfaite ou presque (excepté un cas) pour Es-Epa (Chi-square test), Es-Fin, Epa-Fin, Es/Epa-Fin. Ceci pourrait laisser penser qu'un progrès de l'état somatique (diminution de la fréquence ou de l'intensité) est le signe prédictif d'une probable amélioration de l'état psychique et aussi de ce qui est considéré comme une réussite de la prise en charge. En effet, la première demande des patientes est bien focalisée sur le symptôme somatique, mais l'élargissement psychique proposé fonctionne. Par contre, on ne retrouve pas les mêmes liens entre ces variables et l'auto-évaluation des patientes. Cette faible population ne permet pas de faire des hypothèses à ce stade. La question reste donc posée autant pour les facteurs externes que pour l'ensemble des variables avec le bénéfice des CCMP pour les patientes (PP; auto-évaluation).

Enfin, malgré le nombre réduit de patients, et sans oublier que tout lien pourrait être un pur hasard, il semble néanmoins que les variables Es et Epa puissent être considérées comme pertinentes et correspondre aux exigences de départ, comme par ailleurs les variables U et CM. L'analyse des 
questionnaires montre qu'il s'agit d'une population anxieuse et/ou dépressive, avec peut-être un pourcentage supérieur d'anxiété. La description des PBMI peut se faire autant de façon affective que sensorielle, et un léger penchant pour le côté affectif semble prédominer, ce qui pourrait être l'indice d'une expression psychologique de la gêne. Enfin, les changements psychosociaux peuvent être positifs, négatifs ou neutres.

En résumé, les questionnaires sont pertinents pour une évaluation avant ou après traitement, car ils sont suffisamment sensibles dans chacune de leurs catégories.

\section{CONCLUSION}

La majorité des patientes ayant bénéficié des CCMP semble présenter une amélioration de leur

médecine buccale chirurgie buccale

VOL. $12, \mathrm{~N}^{\circ} 1$ 2006

page 38

\section{RÉFÉRENCES}

1 - BOtha PJ, VAN DER BIJL P, VAN EYK AD. A literature review and pilot study to characterise the treatment of burning mouth syndrome. S Afr Dent J 2001 ; 56: 353-8.

2 - Bouvard M, Cottraux J. Protocoles et échelles d'évaluation en psychiatrie et en psychologie. Masson, Paris, 2000.

3 - BRUCHON-SCHWEITZER M, QUINTARD B (sous la direction de). Personnalité et maladies : stress, coping et ajustement. Dunod, Paris, 2001.

4 - DegIVE DE SAUSSURE C. Somatisation et consultations conjointes médico-psychologiques. Med Hyg 2003 ; 61: 292-6.

5 - Degive de Saussure C, Cunier-Drocco C. Une patiente me dit, pour la énième fois : " ma langue me brûle, je perçois un mauvais goût dans ma bouche... : que puisje faire? Méd Hyg 2003 ; 2424: 359.

6 - Degive C, Guyot J.PH. Les 6 principes de base des consultations conjointes. Otorhinolaryngol Nova 2000 ; 10: 11-5.

7 - Demange C, Husson C, Poï-Vet D, Escande JP. Paresthésies buccales psychogènes «PBP » et dépression : une approche psychanalytique. Rev Stomatol Chir Maxillofac 1996 ; 97: 244-52.

8 - Drocco C, Degive C, Archinard M, SAmson J. Paresthésies buccales médicalement inexpliquées. Réalités Clin 1999; 10: 447-54. état somatique et de leur état psychique. Elles ont également mieux compris les PBMI et sont moins inquiètes. II faut cependant préciser que le petit nombre de patientes inclus dans cette étude ne permet pas de donner des réponses statistiquement significatives. Néanmoins, la pertinence des variables investiguées pendant l'entretien de follow-up semble bonne et permet d'envisager la mise en route à une plus grande échelle d'une évaluation de l'utilité des CCMP dans la prise en charge des PBMI. On doit noter que la fonction «de rassurer et d'expliquer » mériterait de faire l'objet d'un critère spécifique. Enfin, il paraît important de continuer à comparer autoévaluation et hétéro-évaluation des CCMP afin de se rapprocher toujours plus des besoins des patientes.

9 - Drocco C. Patient somatisant et paresthésies buccales médicalement inexpliquées : concept et prise en charge. Thèse en Médecine Dentaire $n^{\circ}$ 606, Faculté de Médecine de l'Université de Genève, 2000.

10 - FIRAS AL, QURAN AM. Psychological profile in burning mouth syndrome. Oral Surg Oral Med Oral Pathol Oral Endod 2004 ; 97: 339-44.

11 - GRUSHKA M, EPSTEIN JB, GORSKY M. Burning mouth syndrome. Am Fam Physician 2002 ; 65: 615-20.

12 - LAMEY PJ, LAMB AB. The usefulness of the HAD scale in assessing anxiety and depression in patients with burning sensation. Oral Surg Oral Med Oral Pathol $1989 ; 67: 390-2$

13 - ROJo L, SilvestRe FJ, De VInCEnte T. Prevalence of psychopathology in burning mouth syndrome. A comparative study among patients with and without psychiatric disorders and controls. Oral Surg Oral Med Oral Pathol 1994 ; 78: 312-6.

14 - ZAKRZEWSKA JM, GLENNY AM, FORSSELL H. Interventions for the treatment of burning mouth syndrome (Cochrane Review): The Cochrane Library 2002 ; 2.

15 - ZIGMOND AS, SNAITH RP. Hospital anxiety and depression scale. Acta Psychiatric Scand 1983 ; 67: 361-70. 